\title{
Hinchadas o barras bravas en Colombia. Una revisión bibliográfica ${ }^{1}$
}

\author{
Supporters or "barras bravas" in Colombia. \\ A bibliographic review
}

John Alexander Castro Lozano ${ }^{2}$

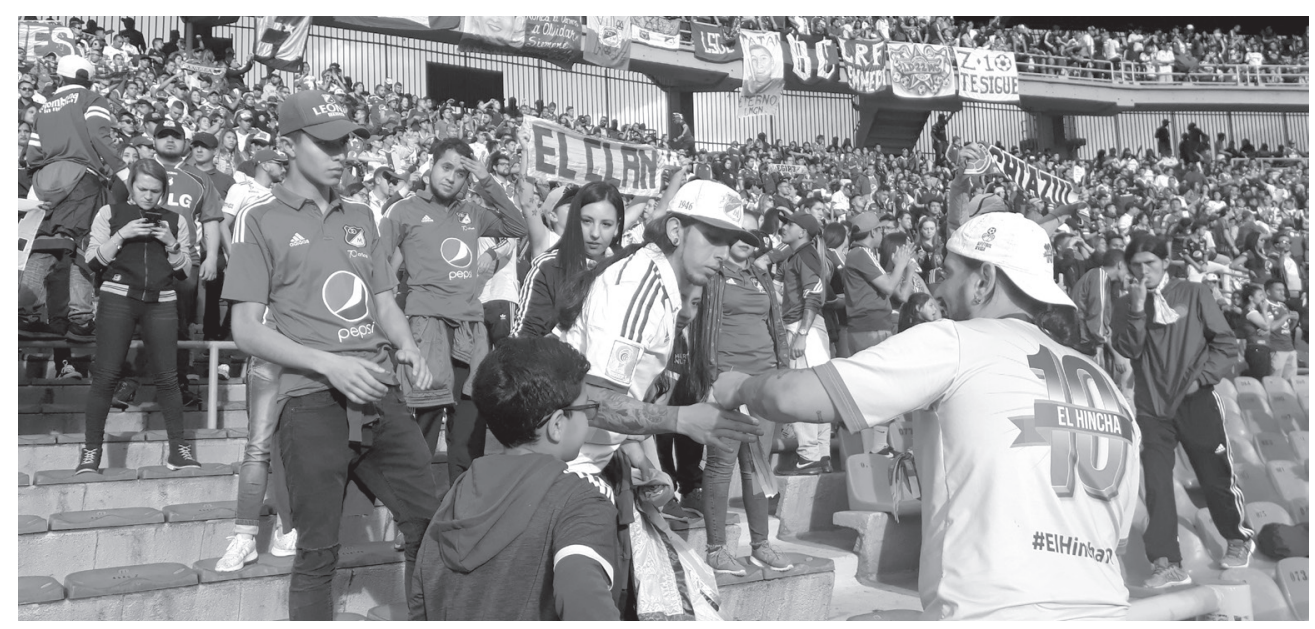

\section{Resumen}

Los aficionados al fútbol se transformaron en el transcurso del siglo XX. Un nuevo rol los llevó a participar activamente en el juego desde las tribunas y "continuar el partido" en las calles. De esta manera, el propósito del artículo es construir una revisión bibliográfica de los estudios publicados por investigadores colombianos, cuyo referente de análisis son las hinchadas o las "barras bravas". Las bases de datos de CLACSO, Dialnet, FLACSO, RedALyC y SciELO fueron consultadas con el propósito de rastrear las referencias bibliográficas. De esta manera, fue seleccionada la bibliografía publicada desde 2001 hasta 2018. Uno de los resultados muestra que las categorías de rituales, violencia, identidad, aguante, escuela, políticas públicas y símbolos son utilizadas con mayor regularidad para comprender y explicar las expresiones y

Citar este artículo como: Castro Lozano, J. A. (2018). Hinchadas o barras bravas en Colombia. Una revisión bibliográfica. Revista Papeles, 10(20), 90-100.

Fecha de recibido: agosto 15 de 2018 • Fecha de aceptación: noviembre 5 de 2018

1 Este artículo es un avance del proyecto de investigación Yo soy azul y azul seré hasta que me muera, integrado a la línea en Subjetividades, Diferencias y Narrativas -en el marco de la elaboración de mi tesis de grado- en el Doctorado en Estudios Sociales en la Universidad Distrital Francisco José de Caldas.

2 Doctorando en Estudios Sociales en la Universidad Distrital Francisco José de Caldas, Magíster en Estudios Sociales de la Universidad Pedagógica Nacional y Sociólogo de la Universidad Nacional Colombia. Además, es profesor e investigador en la Universidad Antonio Nariño (sede Bogotá) e integrante de los grupos de investigación Conciencia y Vivencias; así como profesor ocasional en la Universidad Pedagógica Nacional. Correo electrónico: alexandercastro1981@gmail.com ORCID iD: orcid.org/0000-0001-8397-7690 
los comportamientos de los hinchadas o barras bravas. Finalmente, los hinchas, en las hinchadas o las barras bravas, construyen formas de relacionarse a partir de vivencias comunes, constituyentes de semejanzas y diferencias en el contexto del fútbol.

Palabras clave: fútbol, hinchas, hinchadas, barras bravas, revisión bibliográfica.

\begin{abstract}
Football fans were transformed in the course of the twentieth century. A new role led them to actively participate in the game from the stands and "continue the game" on the streets. In this way, the purpose of the article is to build a bibliographic review of the studies published by Colombian researchers, whose benchmark of analysis is the supporters or the "barras bravas". The databases of CLACSO, Dialnet, FLACSO, RedALyC and SciELO were consulted with the purpose of tracking bibliographic references. In this way, the bibliography published from 2001 to 2018 was selected. One of the results shows that the categories of rituals, violence, identity, endurance, school, public policies and symbols are used more regularly to understand and explain the expressions and behaviors of supporters or "barras bravas". Finally, the fans, in the "barras bravas", build ways of relating from common experiences, constituents of similarities and differences in the context of football.
\end{abstract}

Key words: football, fans, supporters, "barras bravas", bibliographic review.

\title{
Introducción
}

Durante la década de 1990, en Colombia se organizaron las barras bravas en ciudades como Bogotá, Cali, Medellín, Barranquilla, Manizales, Bucaramanga, Neiva, Pereira e Ibagué y expusieron renovadas formas de apoyar a los clubes de fútbol. Además, nuevas maneras de identificar a los adversarios futbolísticos. En ese ambiente e iniciaron las publicaciones sobre los sujetos y los grupos sociales en el contexto del fútbol, es decir, sobre los hinchas, las hinchadas y las barras bravas. Desde 2001 hasta el 2018 es posible localizar múltiples publicaciones entre artículos, capítulos de libros y libros, derivados de investigaciones hechas durante carreras de grado o posgrados, otros son resultados de proyectos respaldados por Instituciones de Educación Superior (IES) o entidades gubernamentales. No obstante, en esta revisión bibliográfica no se tendrán en cuenta ya que son un número indeterminado de trabajos o tesis de grado sin publicar que reposan en las bibliotecas de distintas instituciones universitarias colombianas y extranjeras, en los niveles de grado, especialización, maestría o doctorado.
La siguiente gráfica expone, por años, las referencias bibliográficas publicadas por investigadores colombianos. En la gráfica se puede observar que la publicación de los trabajos de investigación, relacionados con las hinchadas o barras bravas es relativamente reciente. Además, el interés por interpretar y comprender los comportamientos o las conductas de los hinchadas o barras bravas se incrementado de forma significativa. De esta manera, el propósito del artículo es elaborar una revisión bibliográfica de diferentes resultados de investigación publicados en Colombia.

Las investigaciones han sido elaboradas por antropólogos, comunicadores sociales, pedagogos, psicólogos, sociólogos, trabajadores sociales e, incluso, desde la experiencia de los hinchas. De este modo, el abordaje se ha realizado desde diversas perspectivas disciplinares, teóricas, conceptuales, metodológicas y técnicas, permitiendo una variedad y riqueza en los resultados de investigación. En los textos, la denominación que se asigna a los 
Tabla 1. Referencias bibliográficas localizadas

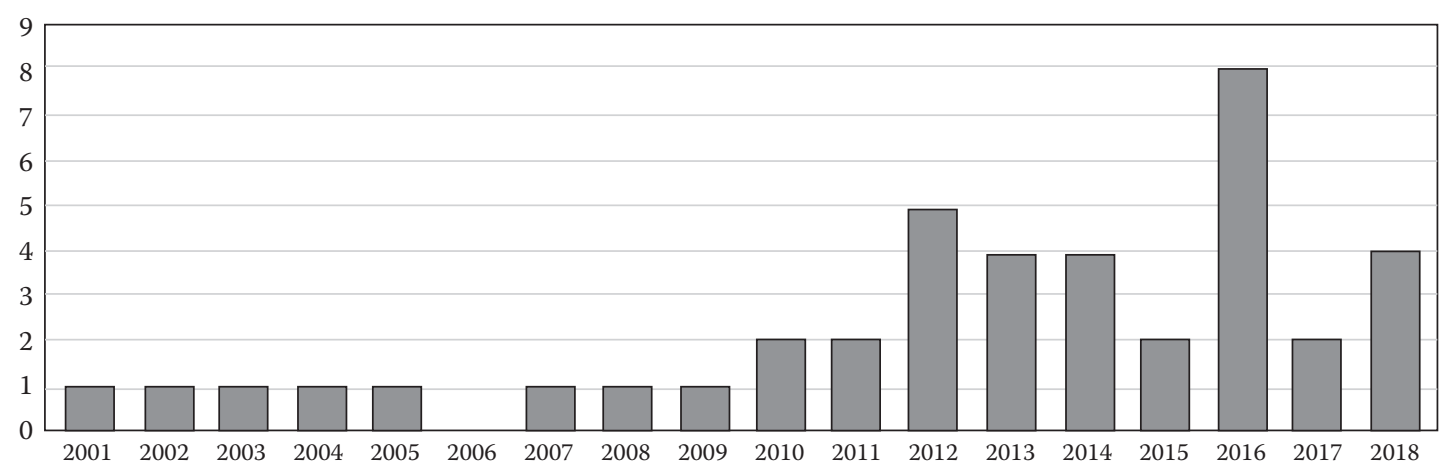

Fuente: propia.

sujetos y a los grupos sociales (en el contexto del fútbol) es heterogénea, pues se los llama barras, barras bravas, barras de fútbol, barras futboleras, barras intensas, barras populares o barras ultras. Y a los sujetos que los forman, los denominan barras, barristas, foforos o hinchas. Las designaciones dependen del interés y la posición de cada uno de los investigadores frente a los sujetos y a los grupos sociales. De este modo, la clasificación de los textos se organiza de la siguiente manera: rituales, violencia, identidad, aguante, escuela, políticas públicas y símbolos. A continuación, se presentan otras revisiones bibliográficas, elaborados por distintos investigadores.

\section{Otros balances bibliográficos}

El estado de la cuestión o antecedentes presentados en esta temática son complementados con otros, pues el propósito es "lograr balances e inventarios bibliográficos para dar cuenta del estado de conocimiento actual sobre un concepto" (Gómez, Galeano, \& Jaramillo, 2015, p. 427). En este sentido, Hernández, Molina, Pérez, Colomo, Gálvez y Ortega (2001) resaltan los aportes y las críticas del Grupo de Leicester, liderado por Norbert Elias y Eric Dunning (desde la perspectiva del proceso civilizatorio), apoyados por Patrick Murphy y John Williams; la reflexión de los desastres colectivos de David Canter, Miriam Comber y David Uzzel; el análisis sobre el vandalismo en el fútbol de Javier Durán; el modelo psicosocial de Jhon Kerr y, por último, el estudio del comportamiento colectivo de Federico Javaloy.

Vieira y Siqueira (2008) recopilan distintos trabajos de investigación a partir de los siguientes criterios: están relacionados con la problemática de la violencia en los estadios de fútbol y la participación de las torcidas organizadas. Esos trabajos de investigación caracterizan y describen el problema; el análisis se construye a partir de los discursos y los testimonios de hinchas, periodistas, juristas, policías y autoridades, desde sus experiencias en el contexto de la violencia. Aponte, Pinzón, Rodríguez y Vargas (2009) señalan que la producción bibliográfica ha sido realizada por antropólogos, sociólogos y psicólogos. En ese aspecto, destacan que los trabajos antropológicos enfatizan en el análisis de los símbolos, los códigos y las representaciones; las reflexiones sociológicas acentúan en los procesos de construcción identitaria y los estudios psicológicos destacan la comprensión de las conductas violentas y las manifestaciones de agresividad. Además, realizan algunas anotaciones sobre los programas, la normatividad y el cubrimiento hecho por los medios de comunicación en Colombia.

Castro (2010) recoge diferentes resultados de investigación realizados desde el trabajo de campo etnográfico y los ordena a partir de las nociones de identidad, práctica ritual y aguante. Identidad y práctica ritual son 
conceptos utilizados por distintas disciplinas de las ciencias sociales y el aguante es una noción y una práctica utilizada por las denominadas hinchadas o barras bravas que les permite distinguirse de otros hinchas y de grupos semejantes. Lopes y Cordeiro (2010) elaboran una presentación sobre los orígenes y las características de las torcidas organizadas; plantean las semejanzas con las barras bravas y los hooligans y, presentan las particularidades en relación con estos grupos. Por su parte, Alabarces (2013) destaca las reflexiones de los ingleses Ian Taylor, Oliver Popplewell, Eric Dunning, Patrick Murphy, John Williams, Gary Armstrong y Richard Giulianotti. Y en Argentina resalta los trabajos pioneros de Amílcar Romero y Eduardo Archetti, continuadas con las investigaciones de Pablo Alabarces, José Garriga y Verónica Moreira, a partir del concepto del aguante. Además, repasa la intervención estatal para enfrentar la violencia en el fútbol en Inglaterra y Argentina.

Guedes (2013) organiza su artículo en dos momentos: primero, estudia la bibliografía sobre las torcidas organizadas $y$, segundo, analiza la contribución de esa bibliografía en la investigación etnográfica sobre el control ejercido por la policía. En este sentido, expone los comportamientos característicos de las torcidas organizadas y las contradicciones presentadas en el ejercicio del control de la policía; contrastando los resultados de su trabajo de campo y las observaciones de la bibliografía consultada. En el artículo de Palhares, Cabrera y Schwartz (2014) se usan los descriptores de ocio, torcidas organizadas, agresividad y violencia como guía de selección bibliográfica. De esta manera, afirman que el comportamiento individual de los hinchas puede cambiar a partir de la composición de la torcida organizada, de la violencia en la sociedad, el contexto sociohistórico y la impunidad, de la influencia de los medios de comunicación y de los problemas en la organización de la competición deportiva. Y Villanueva y Gómez (2018) presentan una revisión bibliografica (de trabajos y tesis de grado de distintas IES) a partir de las categorías de barras bravas, tipologías de violencia en el futbol y procesos de convivencia o políticas públicas. De esta manera, el artículo pretende localizar las posibilidades de la solución de los conflictos alrededor del fútbol.

\section{Las barras de fútbol están conformadas mayoritariamente por jóvenes, su dinámica central es la religiosidad, ya que poseen un ideal sagrado sobre los roles y las expresiones de los hinchas demostrados en cada partido.}

\section{Los rituales entre los hinchas}

Las barras de fútbol están conformadas mayoritariamente por jóvenes, su dinámica central es la religiosidad, ya que poseen un ideal sagrado sobre los roles y las expresiones de los hinchas demostrados en cada partido (Mateus \& Mahecha, 2002). Diego Bolaños (2013) presenta los elementos hallados en una barra en los que se evidencian motivaciones, mitos, rituales, relaciones interpersonales, contextos y redes de agrupaciones juveniles. Esas expresiones de la barra vigorizan las dinámicas citadinas con sus distintos recorridos, reivindicando manifestaciones populares de celebración (carnavales). José Andrade, Andrés Ahumada, Ana Borja, Jennifer Soto y Juan Villarreal (2014) revelan la correspondencia entre el consumo de alcohol y la pertenencia a las barras bravas, porque está relacionado con aspectos socioculturales, rituales, la vida social y la cohesión del grupo. La impulsividad es característica de niveles organizativos bajos. 


\section{La violencia en el contexto del fútbol}

Héctor Lambuley (2003) denomina foforos a los integrantes de las barras intensas, quienes asumen un papel intolerante y violento frente a los hinchas de equipos rivales. El orden interno de las barras intensas les permite organizarse y diferenciarse del público tradicional. La violencia en el fútbol en Colombia, sostiene Jesús Vélez (2005), fue asimilada mediante los sistemas de comunicación, promoviendo las rivalidades y la violencia en los estadios, perjudicando a la juventud y, así, las barras bravas son una perversión. Los hinchas jóvenes reflejan mayor arraigo por su equipo y demuestran mayor fidelidad, fervor y prácticas violentas, afirman Gregorio Morales y Jahen Morales (2011); los hinchas que pertenecen a la barra adquieren prestigio y eligen estar allí "guerreándola" por el equipo.

Clara Gutiérrez, Vivian Hernández, Joan Rodríguez y Andrea Suárez (2012) muestran la correlación positiva alta entre los rasgos de personalidad (psicoticismo y la extroversión) y la conducta antisocial (actos violentos y delictivos) en función de variables sociodemográficas (equipo de fútbol, sexo, edad, estrato y ocupación) en barristas del fútbol. Guillermo Castaño, Nicolás Uribe y Sandra Restrepo (2014) exponen las relaciones de las barras bravas con la violencia y el consumo de sustancias psicoactivas, mostrando el efecto neuropsicológico sobre el comportamiento de los hinchas. Sin embargo, señalan algunas propuestas que posibilitan plantear programas de prevención en los estadios. Jhon Londoño (2015) examina las manifestaciones de las barras ultras: apoyar a su equipo, delimitar su territorio fuera de los estadios, organizar su jerarquía, dividirse en subgrupos, expresar la violencia (simbólica y física) y estar conformada, mayoritariamente, por jóvenes.

Juan Arboleda y Margarita Vélez (2016) identifican diferentes argumentos y discursos usados en prensa periódica que responsabilizan a un "hincha" indefinido o cualquiera, particularizando su comportamiento, criminalizándolo y estigmatizándolo; estableciendo sensaciones, supuestos e interpretaciones sobre la violencia en el fútbol. El rol de la mujer en el contexto del fútbol es descrito por Eliana Ramírez y Jaime Restrepo (2018), particularmente su participación en la barra brava. Las mujeres son tratadas como integrantes que pueden asumir el ejercicio de la violencia, transformando su estilo, sus costumbres, formas de hablar y sus actitudes, exponiendo características varoniles. Nicolás Uribe (2018) propone una relación entre barras de fútbol y masas psicológicas a partir de los "actos de violencia" y los fenómenos psicológicos como las identificaciones, el contagio o la imitación, comparación que pretende considerar y redefinir las estrategias de intervención, sean legales, psicopedagógicas o políticas represivas.

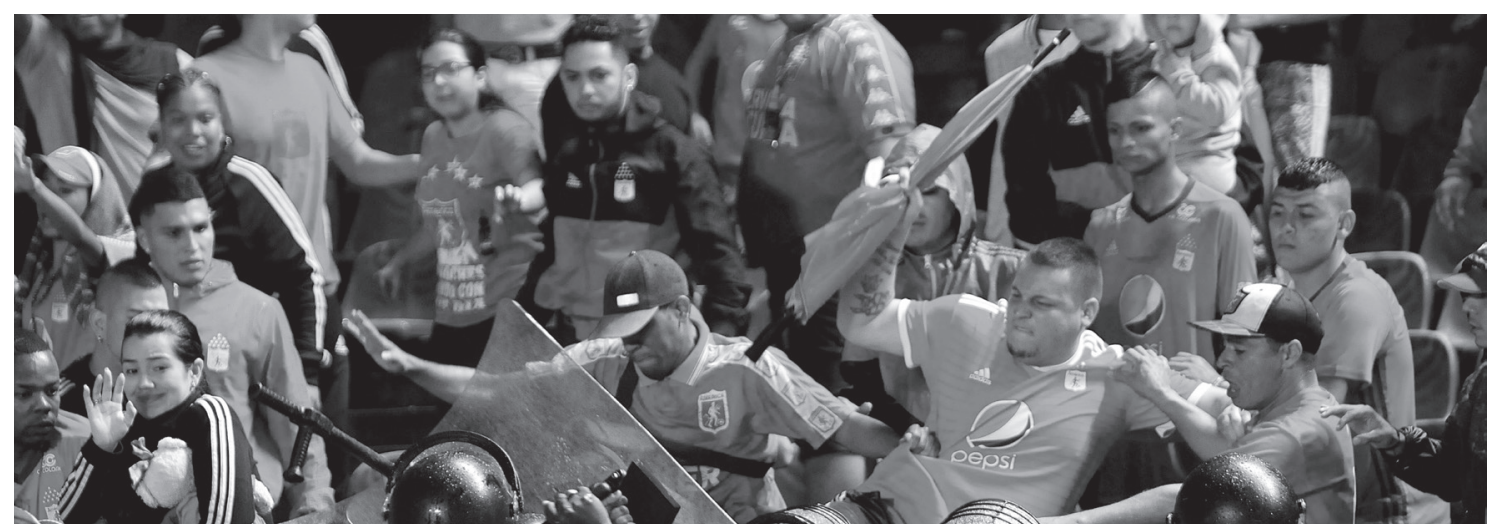

Universidad Antonio Nariño • Facultad de Ciencias de la Educación 


\section{La identidad constituida en el fútbol}

Las barras de fútbol son conformadas por jóvenes, destacan Harold Pardey, Juan Galeano y Andrés Blanco (2001), quienes expresan formas carnavalescas y agresivas al acompañar a su equipo, permitiendo un encuentro pluralista, desbordar las emociones y constituir una identidad mediante la pasión, manifestada en el aguante. María Salcedo y Omar Rivera (2007) desde "las prácticas sociales y su relación simbólica de apropiación del espacio, considerando la imitación de las acciones y la percepción sensorial de las mismas" (p. 24) en la vida cotidiana de las barras bravas, observan la constitución de su identidad. La violencia entre los jóvenes integrantes de las barras bravas, explica Nurys Silva (2010), está relacionada con la constitución de un tipo de masculinidad y con la aceptación de prácticas y formas de identidad, posibilitándoles maneras de interacción social.

La violencia entre las barras bravas es simbólica y física, sostiene Germán Gómez (2011), ya que tienen la intención de agredir o eliminar al rival, fijando las prácticas de los hinchas en torno al aguante como una forma de ratificar su identidad. Oscar Andrade y Diego Walteros (2012) establecen los elementos que determinan la constitución de la identidad y sus particulares formas de interacción en las barras bravas. También identifican las causas que determinan los comportamientos violentos y evalúan la utilidad del programa Goles en $\mathrm{Paz}^{3}$. Catalina López y Yehuda Neumark (2012) identifican los

3 'Goles en Paz' fue el Comité de seguridad y convivencia para los espectáculos de fútbol profesional en Bogotá, instituido a partir del Decreto 164 de 2004 en la alcaldía de Luis Garzón. Luego, el Decreto 455 de 2008 transformó 'Goles en Paz' ya que fue adscrito al Programa Vida Sagrada y Desarme de la Secretaria de Gobierno de la Alcaldía Mayor de Bogotá en el gobierno de Samuel Moreno. 'Goles en Paz' se mantuvo hasta el año 2013, remplazado por la campaña "Estadio Territorio de Paz" en la alcaldía Gustavo Petro y en la alcaldía de elementos que pueden asociar la membresía a las barras bravas y el consumo de drogas, buscando plantear propuestas de reducción, pues la correspondencia entre la construcción de una identidad masculina y la aceptación de comportamientos impulsivos es notoria. Las barras bravas son un fenómeno social urbano, señala César Torres (2012), constructor de procesos identitarios y sentidos de pertenencia en los jóvenes, y muestra que el aliento al equipo implica la exhibición de diferentes símbolos en un ambiente festivo en los estadios.

El funcionamiento discursivo de las pintadas o grafiti, analiza Alfredo Mendoza (2013), de integrantes de una barra brava en la ciudad muestran subjetividades juveniles y resignifican el espacio urbano, constituyendo su memoria colectiva y su identidad, delimitando simbólicamente su territorio. Las representaciones sociales constituidas por los actores comunitarios e institucionales, explica Luisa Miranda, Ingrid Urrego y Diana Vera (2015), vinculan a la barra brava (subcultura juvenil) con la violencia en el fútbol. Aunque los barristas sostienen que la barra brava posibilita la socialización y la formación de una identidad colectiva. El ingreso de los jóvenes a las barras bravas -sostienen Manuel Blázquez, Carol Casas, Nicolás Escobar, Diego Garzón, Germán Moscoso, María Rojas y Laura Romero (2016) - les permite asimilar símbolos, expresiones, actitudes e ideologías; nociones defendidas mediante la violencia, asumiendo una identidad colectiva ya constituida en el fútbol.

Natalia Salinas y Luz Suárez (2016) examinan el uso de la categoría de violencia en el fútbol a partir del concepto de identidad, pues la identidad (individual y grupal) permite entender los sentidos de pertenencia y los vínculos que se generan entre los seguidores del fútbol.

Enrique Peñalosa se desarrolló la campaña "Más fútbol, más vida”. 
La marginalidad influye en la decisión de los jóvenes de pertenecer a las barras bravas, señalan Edgar Galeano, Bettsy Cruz, Jeffrey Cruz, Edward Escovar, Andrés Gómez, Juan Nieto y Luisa Rodríguez (2016), pues los individuos localizan factores que les permiten un estilo de vida, buscar una identidad y socializar entre semejantes. Nataly Giraldo y Jaime Restrepo (2017) afirman que las barras de fútbol poseen características semejantes a las "tribus urbanas", son integradas por jóvenes, tienen particulares formas de comunicarse y de posicionarse en el contexto, poseen rituales y símbolos específicos que los distinguen de otros grupos sociales, constituyendo su identidad.

\section{El aguante de los hinchas}

El concepto de energía, muestra Samuel Ávila (2008), permite explicar formas de sentir entre los hinchas, transformada en aguante, una manera de comprender la generación de la emoción. Los hinchas en las barras bravas alientan (apoyan) a su equipo y enfrentan (verbal y físicamente) a sus rivales, demuestra John Castro (2013), estas manifestaciones son denominadas carnaval y combate, respectivamente; la cuales configuran el aguante, una expresión que implica mantener la voz y los puños en alto. El grafiti barrista, resaltan Kevin Rozo y Juan Rojas (2018), permite visibilizar las relaciones entre las barras de fútbol, particularmente las rivalidades o los conflictos, es decir, manifiestan confrontaciones simbólicas que diversifican los significados del territorio $y$, así, muestran un ejercicio de poder en el espacio habitado, vinculado con el aguante.

\section{Los hinchas en la escuela}

Lesley Rico (2016) contribuye al entendimiento de los sentidos y las relaciones que constituyen los jóvenes pertenecientes a barras populares de fútbol con la escuela y la comunidad a partir de sus prácticas convivenciales y académicas, incluyendo las maneras de manifestarse y las dinámicas de la barra como constituyente identitario. Luz Cañón (2017) analiza la violencia en las instituciones educativas, pues jóvenes escolarizados están vinculados a las barras de fútbol, introduciendo distintas problemáticas en las escuelas. Por eso, propone una explicación de la adhesión de los estudiantes a esos grupos, observando sus procesos de socialización.

\section{La política pública en Colombia}

Alejandro Villanueva y Nelson Rodríguez ${ }^{4}$ (2013) destacan los aspectos positivos de la legislación instituida en Colombia, al prevenir los enfrentamientos entre las barras futboleras y estandarizar las acciones que permitan la seguridad, la evacuación, el transporte y el desplazamiento de las hinchadas. Desde su experiencia como hincha e integrante de una barra popular, Raúl Martínez (2016) cuenta la forma organizativa de la barra a la que pertenece, la elaboración de proyectos sociales y comunitarios y la participación en el proceso de construcción de una política pública. Alirio Amaya $^{3}$ (2016) resalta la necesidad de acatar lo estipulado en la legislación colombiana relacionada con las barras futboleras, alfabetizando a los hinchas e involucrarlos en la elaboración de una política pública que incluya aspectos de convivencia, seguridad y comodidad.

Las barras futboleras deben defender la autonomía en sus decisiones con la responsabilidad (individual y colectiva) que esto supone, plantea César Guzmán (2012), con el propósito de proponer una política menor, como acto de resistencia a los poderes hegemónicos. Yudis López, David Libreros, Zoilo Asprilla y Maritza

\footnotetext{
Fueron funcionarios del programa Goles en Paz.

5 Fue director del programa Goles en Paz de la Alcaldía Mayor de Bogotá y fue el secretario técnico de la Comisión Nacional de Seguridad, Comodidad y Convivencia en el Fútbol del Departamento Administrativo del Deporte, la Recreación, la Actividad Física y el Aprovechamiento del Tiempo Libre, Coldeportes.
} 
Turizo (2014) diseñan estrategias de disminución de los incidentes violentos en los estadios y proponen la implementación de estrategias educativas acordadas con los representantes de las barras bravas, las autoridades civiles y la policía nacional, contribuyendo a la elaboración de una política educativa. Santiago Preciado (2018) identifica las relaciones entre las barras populares y el poder político en Colombia, a partir del accionar social y político de las barras de fútbol, ya que Los Del Sur (barra de Nacional de Medellín) ha logrado maneras de relacionarse con la institucionalidad. De este modo, sugiere que en las políticas públicas es necesario incluir una "normatividad para la regulación y creación de institucionalidad [...] para regularizar la actividad de las hinchadas futbolísticas colombianas y el equilibrio en sus relaciones de poder político" (p. 203).

\section{Los símbolos y el contexto del fútbol}

El público se instala en la tribuna, afirma Juan Rivera (2004), lo que le permite manifestar distintos elementos simbólicos y así, presenciar los partidos de fútbol. El autor clasifica el público en espectadores, hinchas y futboleros (fanáticos). Los hinchas y fanáticos se pueden integrar a una barra brava. La vida social y los hábitos del barrista en el fútbol y en la barra brava son descritos por William Zambrano (2014), quien explica sus símbolos, sus relaciones sociales, sus formas de inclusión y de expresión, sus acciones, sus manifestaciones y sus prácticas sociales.

Jairo Clavijo (2010) expone las dimensiones sociales del lenguaje (verbal y no verbal) desde las experiencias de los barristas, al manifestar pertenencia y representación grupal o territorial. Así, las acciones de la barra de fútbol son entendidas en su contexto. Las condiciones y las representaciones de los jóvenes barristas, señalan Víctor Durán, Ramiro Álzate y Sergio Martínez (2016), muestran la relación con la barra y su contexto. Igualmente, destacan que la barra les otorga un sentido a sus vidas y sus prácticas son parte de su cotidianidad. Ángela Londoño y Victoria Pinilla (2009) comprenden "los procesos y trayectorias de configuración de las prácticas ciudadanas y políticas" (p. 74) relacionados con los modos de estar juntos y con otros en un grupo de hinchas que pertenecen a una barra de fútbol.

\section{A modo de conclusión}

La variedad de trabajos sobre los sujetos y los grupos sociales en el contexto del fútbol es amplia y, además, diversa. Por lo tanto, es posible considerar el asunto de los sujetos y los grupos sociales en el contexto del fútbol como un problema de investigación o, por lo menos, de interés académico en el ámbito de los estudios sociales, culturales o jurídicos de Colombia. Sin embargo, la multiplicidad de perspectivas disciplinares, teóricas, conceptuales y metodológicas dificultan su organización o tematización, pero realmente no es un inconveniente.

Lo que sí puede representar un limitante es la instrumentalización de las categorías o las problemáticas sociales, pues son usadas con el objetivo de indagar en la forma, la particularidad, la incidencia o su funcionamiento entre los hinchas y su grupo social. En otras palabras, son bastante reducidos los trabajos investigativos que se ocupen de interpretar a los sujetos y a los grupos sociales en el contexto del fútbol desde las vivencias y los relatos de los sujetos en los grupos sociales. Adicionalmente, la investigación sobre los hinchas en las barras bravas es fértil, pero requiere continuidad, pues no ha sido posible consolidar o mantener un grupo de trabajo que estudie detenidamente esa problemática de las sociedades contemporáneas y los procesos de transformación de los hinchas y las barras bravas. 


\section{Referencias}

Alabarces, P. (2013). La violencia, la academia y el fracaso. En J. Garriga, Violencia en el fútbol: Investigaciones sociales y fracasos políticos (pp. 21-38). Buenos Aires: Ediciones Godot.

Amaya, A. (2016). Leyes, realidades y desafíos. En R. Soto, \& O. Fernández, ¿Quién raya la cancha? Visiones, tensiones y nuevas perspectivas en los estudios socioculturales del deporte en Latinoamérica (pp. 177-190). Buenos Aires: Consejo Latinoamericano de Ciencias Sociales.

Andrade, J., Ahumada, A., Borja, A., Soto, J., \& Villarreal, J. (2014). Niveles de impulsividad y riesgo de alcoholismo en personas pertenecientes a barras bravas en la ciudad de Ibagué-Colombia. Revista Colombiana de Ciencias Sociales, 5(1), 23-38.

Andrade, O., \& Walteros, D. (2012). ¿Desadaptados o sujetos colectivos? Identidad, violencia y paz en las barras bravas. En B. Arteaga, D. Walteros, \& O. Andrade, Identidades, enfoque diferencial y construcción de paz (pp. 313-350). Bogotá: Universidad Jorge Tadeo Lozano.

Aponte, D., Pinzón, D., Rodríguez, D., \& Vargas, A. (2009). Las barras de fútbol en Colombia: Balance de la producción académica y algunas reflexiones sobre su cubrimiento periodístico, programas y normatividad (2000-2008). Bogotá: Centro de Recursos para el Análisis de Conflictos / American Friends Service Committee.

Arboleda, J., \& Vélez, M. (2016). Construcción de la violencia en el fútbol: la psicologización del hincha. Quaderns de Psicologia, 18(2), 71-81.

Ávila, S. (2008). Laterales. Recuperado el 9 de Junio de 2019, de Revista Virtual Universidad Católica del Norte: http://revistavirtual. ucn.edu.co/index.php/RevistaUCN/article/ viewFile/124/242
Blázquez, M., Casas, C., Escobar, N., Garzón, D., Moscoso, G., Rojas, M., \& Romero, L. (2016). Análisis de la falta de identidad como un factor determinante en la integración de los jóvenes a las barras bravas de la ciudad de Bogotá. Papeles, 7(14), 55-64.

Bolaños, D. (2013). Mitos y rituales en la tribu urbana Barón Rojo Sur barra seguidora del Club de fútbol América de Santiago de Cali. Recuperado el 9 de Junio de 2019, de Revista Borromeo: http://borromeo.kennedy.edu.ar/ Artculos/Bola\%C3\%B1osbaronrojosur.pdf

Cañón, L. (2017). Violencia escolar relacionada con barras del fútbol en Bogotá. Bogotá: Universidad Distrital Francisco José de Caldas.

Castaño, G., Uribe, N., \& Restrepo, S. (2014). Barras bravas en el fútbol: consumo de drogas y violencia. Medellín: Fundación Universitaria Luis Amigó.

Castro, J. (2010). Etnografías de hinchadas en el fútbol: Una revisión bibliográfica. Maguaré(24), 131-156.

Castro, J. (2013). El carnaval y el combate hacen el aguante en una barra brava. Revista Colombiana de Sociología, 36(1), 77-92.

Clavijo, J. (2010). Cantar bajo la anaconda: un análisis sociocultural del barrismo en el fútbol. Bogotá: Editorial Pontificia Universidad Javeriana.

Durán, V., Álzate, R., \& Martínez, S. (2016). Mirada sobre el joven barrista y la convivencia ciudadana. Lúdica pedagógica(23), $31-42$.

Galeano, E., Cruz, B., Cruz, J., Escovar, E., Gómez, A., Nieto, J., \& Rodríguez, L. (2016). Barras bravas: ¿Realización desde la marginalidad y las falencias académicas? Una aproximación desde el sujeto como decisor. Papeles, 7(14), 31-41.

Giraldo, N., \& Restrepo, J. (2017). Construcción de identidad en jóvenes de Manizales 
vinculados a barras de fútbol. Ánfora, 24(42), 165-187.

Gómez, G. (2011). Las barras bravas. Un acercamiento sociológico a un fenómeno urbano. Lúdica pedagógica, 2(16), 56-61.

Gómez, M., Galeano, C., \& Jaramillo, D. (2015). El estado del arte: una metodología de investigación. Revista Colombiana de Ciencias Sociales, 6(2), 423-442.

Guedes, S. (2013). Maçaranduba neles! Torcidas organizadas e policiamento no Brasil. Revista Tempo, 19(34), 81-94.

Gutiérrez, C., Hernández, V., Rodríguez, J., \& Suárez, A. (2012). Relación entre rasgos de personalidad y conducta antisocial en función de variables sociodemográficas de un grupo de barristas de fútbol. Cuadernos Hispanoamericanos de Psicología, 12(1), 37-52.

Guzmán, C. (2012). Por una transfiguración de las subjetividades barristas. El barrismo social como multiplicidad emergente en los estadios colombianos. En D. Quitian, Estudios Socioculturales del Deporte. Desarrollos, tránsitos y miradas (pp. 171-177). Armenia: Kinesis.

Hernández, A., Molina, M., Pérez, G., Colomo, A., Gálvez, P., \& Ortega, I. (2001). La violencia en el fútbol: una reseña bibliográfica. Recuperado el 9 de Junio de 2019, de Lecturas: Educación Física y Deportes: http:// www.efdeportes.com/efd29/violenc.htm

Lambuley, H. (2003). Manifestaciones violentas de los aficionados al futbol profesional en Bogotá, D.C. Bogotá: Universidad de Ciencias Aplicadas y Ambientales.

Londoño, Á., \& Pinilla, V. (2009). El barrismo social de Hinchas por Manizales. Una práctica política y ciudadana. Revista Austral de Ciencias Sociales(16), 73-88.

Londoño, J. (2015). Juventud sin oportunidades. Las barras ultras: un paradigma de indolencia estatal. Armenia: Kinesis.

Lopes, F., \& Cordeiro, M. (2010). Torcidas organizadas do futebol brasileiro: singularidades e semelhanças com outros grupos de torcedores da América do Sul e da Europa. Revista Espaço Acadêmico(104), 75-83.

López, C., \& Neumark, Y. (2012). Membresía a barras bravas y su influencia en el uso de drogas. Revista Peruana de Medicina Experimental y Salud Pública, 29(1), 21-27.

López, Y., Libreros, D., Asprilla, Z., \& Turizo, M. (2014). Intervención educativa orientada a contrarrestar la violencia en el estadio Metropolitano "Roberto Meléndez" en Barranquilla, Colombia. Escenarios, 12(2), 35-52.

Martínez, R. (2016). Los del Sur: organización, trabajo social, comunitario y participación en política pública. En R. Soto, \& O. Fernández, ¿Quién raya la cancha? Visiones, tensiones y nuevas perspectivas en los estudios socioculturales del deporte en Latinoamérica (págs. 137-157). Buenos Aires: Consejo Latinoamericano de Ciencias Sociales.

Mateus, G., \& Mahecha, V. (2002). Hacia una interpretación etnográfica de una barra de fútbol. Folios(16), 36-47.

Mendoza, A. (2013). El graffiti y la consolidación de la identidad, y el imaginario del territorio, de los jóvenes de la barra brava Rebelión Auriverde Norte. En Y. Pedraza, \& O. Pulido, Memorias Congreso de Investigación y Pedagogía (pp. 2359-2368). Tunja: Universidad Pedagógica y Tecnológica de Colombia.

Miranda, L., Urrego, I., \& Vera, D. (2015). Barra brava, cultura, violencia y sociedad: el mundo barrista como representación. Prospectiva. Revista de Trabajo Social e Intervención Social(20), 163-191.

Morales, G., \& Morales, J. (2011). 'Guerrearla'. Algunas características de la nueva generación de hinchas del fútbol espectáculo. Una mirada a través del tiempo. Recuperado el 9 de Junio de 2019, de Lecturas: Educación Física y Deportes: http://www.efdeportes. com/efd158/caracteristicas-de-hinchas-delfutbol-espectaculo.htm 
Palhares, M., Cabrera, N., \& Schwartz, G. (2014). Apuntes para un estudio comparativo entre torcidas organizadas e hinchadas. Movimento, 20(Número especial), 163-176.

Pardey, H., Galeano, J., \& Blanco, A. (2001). La ciudad de los fanáticos. Aproximación al fenómeno de las barras de fútbol locales Barón Rojo Sur y Frente Radical Verdiblanco entre los años 1999-2001. Cali: Universidad del Valle.

Preciado, S. (2018). Las barras de fútbol y el poder político en Colombia. Forum. Revista Departamento de Ciencia Política(14), 185-205.

Ramírez, E., \& Restrepo, J. (2018). El rol de la mujer: una perspectiva sociocultural en el fenómeno del fútbol. Revista Ánfora, 25(44), 109-126.

Rico, L. (2016). La construcción de sentido del joven barrista en la escuela en la ciudad de Bogotá. Bogotá: Instituto Latinoamericano de Altos Estudios.

Rivera, J. (2004). La tribuna como otro terreno de juego. Recuperado el 9 de Junio de 2019, de Lecturas: Educación Física y Deportes: http://www.efdeportes.com/efd69/tribuna. htm

Rozo, K., \& Rojas, J. (2018). Barras de fútbol, aguante y luchas territoriales en la ciudad: tipificación del graffiti barrista. Recuperado el 9 de Junio de 2019, de Lecturas: Educación Física y Deportes: http://www.efdeportes.com/index.php/EFDeportes/article/ view/19/227

Salcedo, M., \& Rivera, Ó. (2007). Emoción, control e identidad: las barras de fútbol en Bogotá. Bogotá: Instituto Colombiano de Antropología e Historia.

Salinas, N., \& Suárez, L. (2016). La identidad como categoría teórica para el estudio de la violencia en el fútbol. Revista Criterios, 23(1), 335-349.
Silva, N. (2010). Entre el juego y violencia. Un estudio de caso sobre la agresión entre los jóvenes de las "barras bravas" de Bogotá. Recuperado el 9 de Junio de 2019, de Archive ouverte en Sciences de l'Homme et de la Société: https://halshs.archives-ouvertes.fr/ halshs-00496208/document

Torres, C. (2012). Si no se vive, no se sabe lo grande que es. Metodología aplicada a un estudio de barras bravas. En M. Jimeno, S. Murillo, \& M. Martínez, Etnografías contemporáneas. Trabajo de campo (pp. 277-308). Bogotá: Universidad Nacional de Colombia.

Uribe, N. (2018). Violencia, psicología de masas y barras de fútbol. Revista Criminalidad, 61(1), 85-96.

Vélez, J. (2005). La violencia en el espectáculo del fútbol, una realidad en el nuevo contexto colombiano. Ponencia presentada en el X Congreso Mundial de Historia del Deporte. Sevilla: 2, 3, 4 y 5 de Noviembre.

Vieira, R., \& Siqueira, G. (2008). O problema da violência nos estádios e as torcidas organizadas de times de Futebol: um ensaio teórico sobre importante fenômeno urbano da atualidade. Cad Saúde Colet, 16(1), 21-34.

Villanueva, A., \& Gómez, G. (2018). Hinchadas, barras bravas y fútbol colombiano. Perspectivas críticas desde las investigaciones sociales. Recuperado el 9 de Junio de 2019, de Revista de Ciencias Sociales: http://www. revistacienciasociales.cl/ojs/index.php/ publicacion/article/view/112/94

Villanueva, A., \& Rodríguez, N. (2013). Aspectos legales, jurídicos y normativos sobre barras futboleras en Bogotá y Colombia. En J. Garriga, Violencia en el fútbol: Investigaciones sociales y fracasos políticos (pp. 253-264). Buenos Aires: Ediciones Godot.

Zambrano, W. (2014). Tras las barras bravas. Prácticas comunicativas, identidad y cultura. Bogotá: ECOE Ediciones. 